\title{
A Voronoi-diagram analysis of the microstructures in bulk-molding compounds and its correlation with the mechanical properties
}

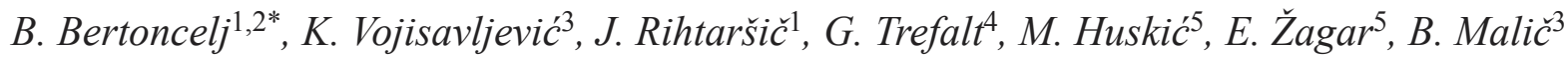 \\ ${ }^{1}$ Domel, d.o.o., Otoki 21, 4227 Železniki, Slovenia \\ ${ }^{2}$ Jožef Stefan International Postgraduate School, Jamova cesta 39, 1000 Ljubljana, Slovenia \\ ${ }^{3}$ Jožef Stefan Institute, Jamova 39, 1000 Ljubljana, Slovenia \\ ${ }^{4}$ University of Geneva, Sciences II,Quai Ernest-Ansermet 30, 1205 Geneva, Switzerland \\ ${ }^{5}$ National Institute of Chemistry Slovenia, Hajdrihova 19, 1001 Ljubljana, Slovenia
}

Received 27 October 2015; accepted in revised form 8 January 2016

\begin{abstract}
Voronoi analysis is implemented to assess the influence of fiber content on the microstructure and mechanical properties of bulk-molding compounds containing different weight fractions of E-glass fibers (EGF) (5-20 wt \%). The fiber distribution in the polymer matrix is analyzed by scanning electron microscopy followed by the Voronoi tessellations, radial distribution function and statistical calculations. The experimental results are compared to modelled microstructures. The derived microstructural descriptors allow us to correlate the fiber weight content and the degree of fiber distribution homogeneity with the mechanical properties of EGF-reinforced composites. The distribution of fibers in composites with 10 and $15 \mathrm{wt} \%$ of fibers could be considered as the most homogeneous. This is in a good agreement with the results of the flexural strength and dynamic mechanical analyses, which confirmed that the latter samples exhibit the highest level of reinforcement.
\end{abstract}

Keywords: polymer composites, microstructures, mechanical properties

\section{Introduction}

Bulk-molding compounds are multiphase composite materials, most commonly consisting of a polymer resin as the matrix, discontinuous glass fibers as the secondary phase and mineral filler. BMCs are widely employed in transportation, in the electrical and the electronic industries, especially for the mass production of small, complex-shaped components, since they offer ease of manufacturing, have low costs, and exhibit good mechanical properties [1]. Common processing methods for the production of such parts are injection and compression molding [2]. The mechanical properties of composite components are strongly influenced by the morphology and weight percentage of the glass fibers. Evidently, an inhomogeneous microstructure can result in local variations of the stress concentration, which can affect the mechanical performance of the composite material $[3,4]$.

In recent years, in order to characterize the microstructures of fiber-reinforced composites, different image-analysis techniques have been applied using optical microscopy or scanning electron microscopy (SEM) [5-7]. One of the methods used to quantitatively describe the microstructure is a Voronoi diagram, commonly known as the Dirichlet tessellation $[8,9]$. This approach is often used for describing noncrystalline structures for example amorphous solids, liquids, and dense gases $[10,11]$. This is a method of dividing a plane into areas known as Voronoi poly-

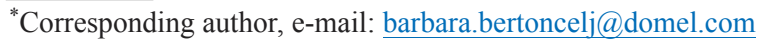
(C) BME-PT
} 
gons. Every polygon is assigned to a given point in a plane and occupies a region around that point, allowing that all neighboring points are closer to the tracked point than to any other point in the set. In fact, a set of convex polygons could be formed by introducing planar cell walls that are perpendicular to the lines connecting neighboring points. Voronoi analysis can be extended also in three dimensions. In this case, the Voronoi cells are defined as 3D polyhedrons $[10,12]$. In material science the Voronoi diagram was used to quantify the distribution of the zirconia phase in alumina-matrix composites [13]. This study pointed out that the wear resistance is related to the microstructural homogeneity of the composite. Trefalt et al. [14] studied the distribution of tetragonal zirconia particles in $\mathrm{Pb}(\mathrm{Zr}, \mathrm{Ti}) \mathrm{O}_{3}$ ceramic composites using a Voronoi-diagram analysis. Summerscales et al. [15] used the Voronoi method to investigate the relationship between the processing, properties and structure in fiber-reinforced polymer-matrix composites. A characterization of sisal-reinforced composite microstructures was carried out by Sun et al. [16]. They characterized the porous structure of the sisal fibers and studied the effect of a maleated polypropylene compatibilizer on the polypropylene matrix's crystallization using Voronoi diagrams. They also used the Voronoi method to perform a quantitative analysis of the fiber distribution within the polymer matrix in order to relate it to the tensile modulus of differently treated composites. Ghosh et al. [17] applied a Voronoi diagram to characterize computer-simulated multiphase microstructures. The same authors also used the radial distribution functions (RDFs) to analyze the patterns in their composites. These RDFs give information about the distribution of particles around a central particle and can therefore be used to detect clustering in the system [17]. Another study of simulated microstructures was made by Pyrz and Bochenek, who investigated the topological disorder of inclusions in composites [18].

On the other hand, microstructural parameters, such as the length, loading, orientation and distribution of the reinforcing fibers, were identified as the most significant factors affecting the mechanical properties of composites. A dynamic mechanical analysis (DMA), where the storage modulus $\left(E^{\prime}\right)$, loss modulus $\left(E^{\prime \prime}\right)$ and damping loss factor $(\tan \delta)$ are measured as a function of temperature and frequency, was found to be useful in correlating the mechanical properties with the microstructure of the material [19-22]. Recently, studies were made of the influence of various glass/natural-fiber volume fractions, such as ramie and curaua fibers, on the dynamic mechanical properties [23, 24].

The aim of this study was to correlate the microstructural features, i.e. inhomogeneous fiber distribution and fiber clustering, with mechanical properties of BMC samples. Such approach could then be applied to obtain insight about mechanical response of critical parts of products manufactured from BMCs, especially those of complex geometry through microstructural analysis.

The Voronoi-diagram method and a RDF analysis were used to quantitatively characterize the microstructures of BMC composites fabricated with different weight fractions of glass fibers in order to evaluate the homogeneity of the fiber distribution in a polymer matrix (PM). The relationship between the homogeneity of the fiber distribution in crosssection, i.e. perpendicular to the compound filling flow direction upon compression molding, and the mechanical properties of the composites was investigated. Therefore, the effect of the glass fiber's weight fraction on the static and dynamic mechanical properties of the composites, such as the flexural strength, storage modulus, loss modulus and damping behavior, is related to the level of the microstructural homogeneity evaluated with the Voronoi analysis and the clustering described by the RDF.

\section{Experimental}

\subsection{Materials}

Four formulations of BMC (Tetradur Gmbh., Hamburg, Germany) were commercially prepared by varying the glass-fiber and mineral-filler weight fractions. As a reference, a composite of polymer matrix (subsequently denoted as PM) and mineral filler was also prepared. The E-glass fibers, denoted as EGF, were $4.5 \mathrm{~mm}$ long, with a diameter of approximately $11 \mu \mathrm{m}$, while the mineral filler was $\mathrm{CaCO}_{3}$ with a particle size range from a few microns up to a few tens of microns as determined by SEM. Furthermore, the PM was based on thermosetting unsaturated polyester, styrene and additives, comprising $21 \mathrm{wt} \%$ of the overall composite material. The compositions of the samples are collected in Table 1. 
Table 1. Compositions of the BMC composite materials

\begin{tabular}{|c|c|c|c|}
\hline No. & $\begin{array}{c}\text { PM } \\
{[\mathbf{w t} \%]}\end{array}$ & $\begin{array}{c}\text { EGF } \\
{[\mathbf{w t} \%]}\end{array}$ & $\begin{array}{c}\mathbf{C a C O}_{3} \\
{[\mathbf{w t} \%]}\end{array}$ \\
\hline 0 & 21 & 0 & 79 \\
\hline 1 & 21 & 5 & 74 \\
\hline 2 & 21 & 10 & 69 \\
\hline 3 & 21 & 15 & 64 \\
\hline 4 & 21 & 20 & 59 \\
\hline
\end{tabular}

The test specimens were fabricated by compression molding, in accordance with the standard ISO 3167 [25]. About $42 \mathrm{~g}$ of the compound was placed into a mold cavity that was heated to around $165^{\circ} \mathrm{C}$ and pressed at a molding pressure of $20 \mathrm{MPa}$ on a hydraulic compression press (HPK 100/6, Bussmann, Munchen, Germany) with a cure cycle of 2 minutes. After molding, the test specimens were cooled down to room temperature. The scheme in Figure 1 indicates the dimensions of the samples and the filling direction of the material during the compressionmolding process. The test specimens were then cut perpendicular to the filling-flow direction, as represented in Figure 1, to yield a cross-sectional area with dimensions of $10 \mathrm{~mm} \times 4 \mathrm{~mm}$.

\subsection{Microstructural characterization}

The cross-section microstructures of the composites were investigated using a field-emission scanning electron microscope FE-SEM (JSM-7600F JEOL Ltd., Tokyo, Japan). Prior to the investigation, special attention was paid to the standard metallographic technique normally used for the preparation of flat surfaces for the SEM investigation in order to establish the best conditions for the grinding and polishing of the samples' cross-sections. The polished

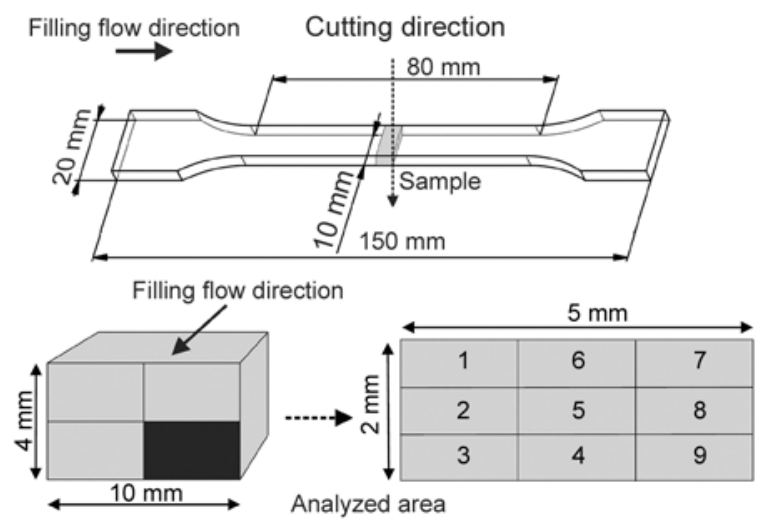

Figure 1. Schematic representation of the test specimen's dimensions, the cutting position and the sample dimensions, with the sampling scheme of the cross-sectional area for the morphological characterization cross-section surfaces were sputter-coated with a thin carbon layer and micrographs were taken in backscattered-electron mode (composition contrast mode - COMPO) at an accelerating voltage of $15 \mathrm{kV}$.

For each sample, the cross-sectional area was divided into quarters, as shown in Figure 1. The sampling included capturing 9 images from 9 segments in one quarter. Each image size was $481 \mu \mathrm{m} \times 361 \mu \mathrm{m}$. The overall investigated area for each sample was $1.6 \mathrm{~mm}^{2}$.

\subsection{Voronoi diagrams}

The Voronoi diagrams were generated from the SEM micrographs. The process included analyzing 9 SEM micrographs taken from 9 different segments of an individual sample, as shown in Figure 1, and proceeded as follows (see Figure 2). First, the center of each glass fiber on the SEM micrograph (a) was marked with a black point using Corel Paint Shop Pro X7 software. The oval-shaped and elongated fibers were treated in the same way, meaning that the point mark was put at the center of the intersection area of each fiber. The point-pattern images (b) were then saved as binary images and subsequently employed to construct the Voronoi polygons (c) using ImageJ software. The area of each Voronoi polygon was measured using Image Tool software. As a reference, for each composition of the composite material, a pattern of randomly distributed disks (d) and a corresponding Voronoi diagram were generated (e). For the randomly generated pattern a constraint of 'no overlapping disk' was used. The randomness of the produced structures was ensured by the use of uniformly distributed random numbers in the $[0,1]$ interval, which were used to generate random positions within the sample. Check of randomness was done by analyzing such point distributions which yielded RDF functions whose value was 1 in the whole interval. This ensures that the points are distributed perfectly random. Furthermore, the area distributions of our randomly generated Voronoi tessellations are in agreement with literature [26]. A sample area and a disk size of $481 \mu \mathrm{m} \times 361 \mu \mathrm{m}$ and $11 \mu \mathrm{m}$ were chosen; these values correspond to the experimental values.

The tessellation of a microstructure into Voronoi polygons is a critical step in generating the geometrical descriptors necessary to quantify the fibers' distribution in a given microstructure of the com- 


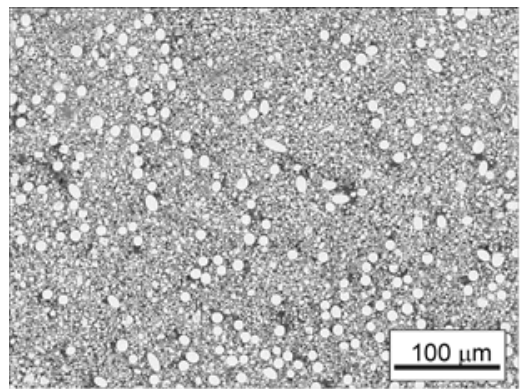

a)

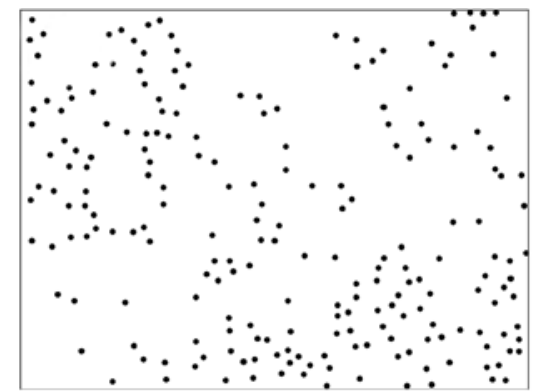

b)

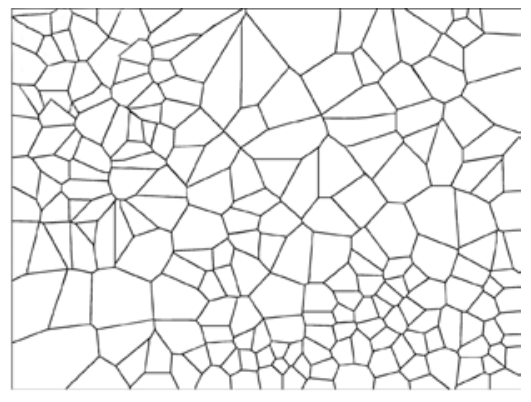

c)

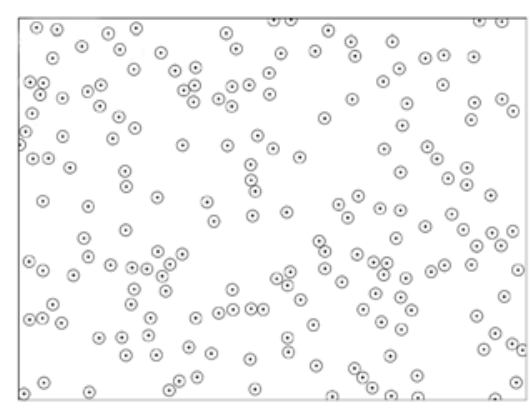

d)

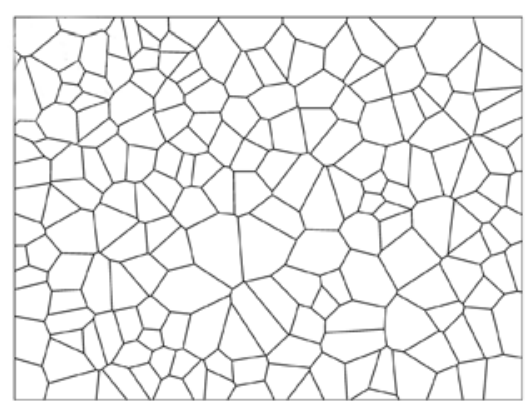

e)

Figure 2. Representation of the sequence of steps involved in generating the Voronoi diagrams from the SEM micrograph (top) and from the randomly distributed disks (bottom): a) SEM micrograph, b) binary point-pattern image obtained from the SEM micrograph by point marking of each fiber, c) the Voronoi diagram generated from the point-pattern image, d) modelled microstructure of random distribution of fibers and e) the Voronoi diagram generated from the modelled microstructure. Note that the minimum interpoint distance in d) was set to be equal to the fibers' diameter, which ensures that there is no overlap of the fibers

posite material. In this way, the analyzed areas 1-9 (see Figure 1) could be easily identified and quantified using statistical parameters like the mean area size of the polygons, the standard deviation and the relative polygon areas. The number of polygons included in the calculations was 468, 998, 1370 and 1979 for the composites with fractions of EGF from 5 to $20 \mathrm{wt} \%$, respectively. Between 250 and 1000 realizations were used in the case of the randomly generated samples to ensure good statistics. The areas of the Voronoi polygons were calculated for each sample. The absolute areas were then normalized to relative polygon areas, which enables an easier comparison of the samples with different fractions of fibers. The relative polygon areas, $A_{\mathrm{r}}$ were calculated according to Equation (1):

$A_{\mathrm{r}}=\frac{A_{\mathrm{p}} N}{A_{\text {total }}}$

where $A_{\mathrm{p}}$ is the absolute polygon area, $N$ is the number of polygons in the sample, $A_{\text {total }}$ is the area of the sample. Such a normalization defines the mean area of the polygons as being equal to 1 , regardless of the fiber fraction. Histograms of the relative polygon areas were generated for each sample.
In addition, the shape of the fibers was obtained from binary (black fiber on a white background) images of the microstructures in which the individual fibers were outlined in black. A stereological analysis was performed using the Image Tool program. The elongation shape factor was determined as the ratio of the longest to the shortest axis of the fiber crosssection.

\subsection{Radial distribution function analysis}

The fiber-reinforced composite microstructures and the randomly created disk patterns were quantitatively described by the RDF, which is related to the probability of finding a particle $j$ at a distance $r$ from the center of a particle $i$, and is defined as Equation (2):

$g_{\mathrm{ij}}(r)=\frac{1}{\rho_{\mathrm{j}} N_{\mathrm{i}}} \sum_{\mathrm{i}} \sum_{\mathrm{j} \neq \mathrm{i}}\left\langle\delta\left(r-r_{\mathrm{ij}}\right)\right\rangle$

where $\rho_{\mathrm{j}}$ is the density of the particles $j, N_{\mathrm{i}}$ is the number of particles $i, r$ is the distance from the center of the particle $i, r_{\mathrm{ij}}$ is the distance between the $i$ and $j$ particles, and $\left\langle\delta\left(r-r_{\mathrm{ij}}\right)\right\rangle$ is the averaged delta function [27]. In fact, the RDF measures the density of the particles $j$ at a distance $r$ from the center of 
the particle $i$, normalized by the average density of the particles $j$ (Equation (3)):

$g_{\mathrm{ij}}(r)=\frac{\rho_{\mathrm{ij}}(r)}{\rho_{\mathrm{j}}}$

where $\rho_{\mathrm{ij}}$ is the local concentration of particles $j$ at a distance $r$ from a particle $i$. For the calculation of the RDFs, periodic boundary conditions with a minimum image convention were used [27, 28].

\subsection{Flexural properties}

The flexural strength of the test specimens was obtained using the three-point bending method with a $64 \mathrm{~mm}$ span and a crosshead speed of $2.0 \mathrm{~mm} / \mathrm{min}$ (Alpha 50-5, Messphysik Materials Testing Gmbh., Fürstenfeld, Austria), in accordance with the standard ISO 178:2003 [29]. The test was carried out under ambient conditions using 15 specimens for each set. The flexural strength was calculated using Equation (4):

$\sigma_{\mathrm{f}}=\frac{3 P l}{2 b h^{2}}$

where $\sigma$ is the flexural strength, $P$ is the maximum fracture load, $l$ is the span between the supports, $b$ is the width of the sample and $h$ is the thickness of the sample.

\subsection{Dynamic mechanical analysis (DMA)}

Dynamic mechanical analyses were performed on a TA Q800 thermal analyzer (Q800 DMA, TA Instruments, New Castle, Delaware, United States) in order to evaluate the dynamic moduli ( $E^{\prime}$ and $\left.E^{\prime \prime}\right)$ and the damping behavior $(\tan \delta)$ of the BMC composites. The $\tan \delta$ is the ratio between loss $\left(E^{\prime \prime}\right)$ and storage modulus ( $E^{\prime}$ ) (Equation (5)):

$\tan \delta=\frac{E^{\prime \prime}}{E^{\prime}}$

For the measurement the test specimens were cut with a diamond blade saw to the length of $60 \mathrm{~mm}$ and thinned to $3.5 \mathrm{~mm}$ by grinding (the width remained the same, i.e. $10 \mathrm{~mm}$ ). The test was carried out at a frequency of $1 \mathrm{~Hz}$ and at strain amplitude of $10 \mu \mathrm{m}$ using a three-point bending mode. The properties were measured in the temperature range $20-200^{\circ} \mathrm{C}$ at a heating rate of $2^{\circ} \mathrm{C} / \mathrm{min}$.

\section{Results and discussion}

\subsection{Microstructure, RDF analysis and Voronoi diagrams}

\subsubsection{Microstructure and Voronoi analysis of a selected cross-sectional segment}

The segment 4 of the overall analyzed positions at the sample's cross-sectional area (see Figure 1) was chosen to represent the microstructure of the composites and the distribution of the EGF in the PM. The microstructures of the polished cross-sections of all the BMC composites with the corresponding Voronoi diagrams, and Voronoi diagrams from randomly generated microstructures are collected in Figure 3. The SEM micrographs show the distributed glass fibers, which are surrounded by irregularly shaped particles of $\mathrm{CaCO}_{3}$ mineral filler and the PM (dark regions). Because the orientation of the glass fibers in the material is random, the intersections of the glass fibers that are perpendicular to the cutting position are circular and others, which are cut at an angle, are oval-shaped.

In order to distinguish between the microstructures with possible fiber clustering and a more even and regular fiber distribution in the composites with different EGF contents, the Voronoi diagrams from our experiment were compared with the diagrams from randomly generated microstructures (see Figure 3, central and right columns). It is clear that at lower weight fractions of the EGF, i.e., at $5 \mathrm{wt} \%$, the Voronoi polygons are quite large. However, by increasing the weight fraction of the EGF the spatial distances between the fibers become smaller, and that is reflected in the smaller polygon areas. In particular, marked differences in the size of the polygons are observed in the Voronoi diagrams of the composites with 15 and $20 \mathrm{wt} \%$ of EGF (Figure $3 \mathrm{c}$, $3 d$ ). In experimental diagrams the relatively small polygon areas indicate EGF clustering within the $\mathrm{PM}$, which arises from the insufficient space for the fibers to orient and regularly distribute at higher weight fractions. In contrast, homogeneous fiber distributions, which are reflected in equally sized polygons across the whole pattern, can be seen in the Voronoi diagrams obtained from the computer-generated random distribution of fibers.

\subsubsection{Voronoi and RDF analyses of the cross-sectional area}

The quantitative description of the fiber distribution in the host PM within the cross-sectional area, as 

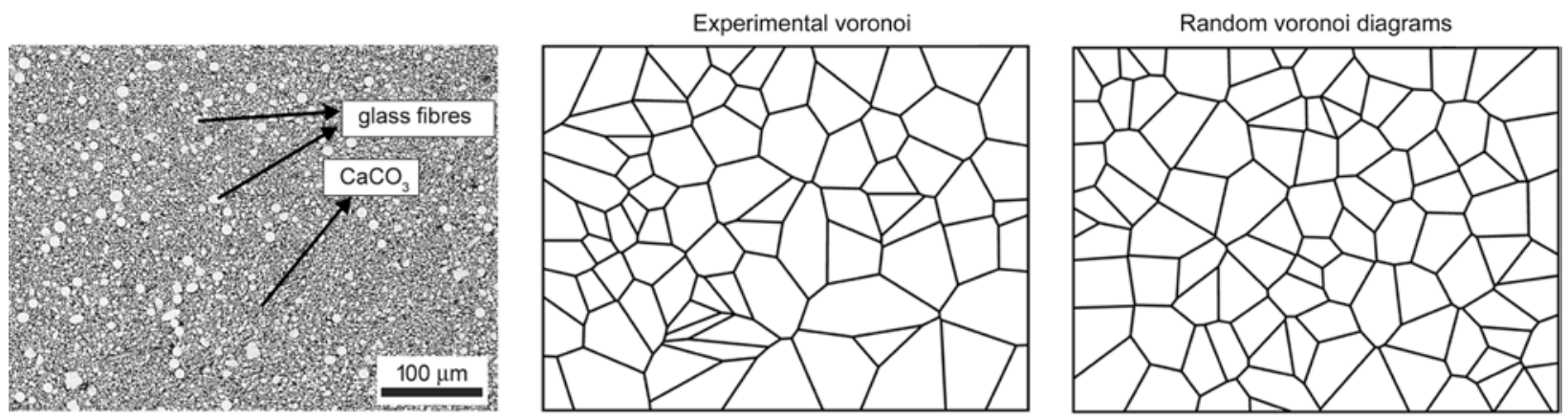

a)
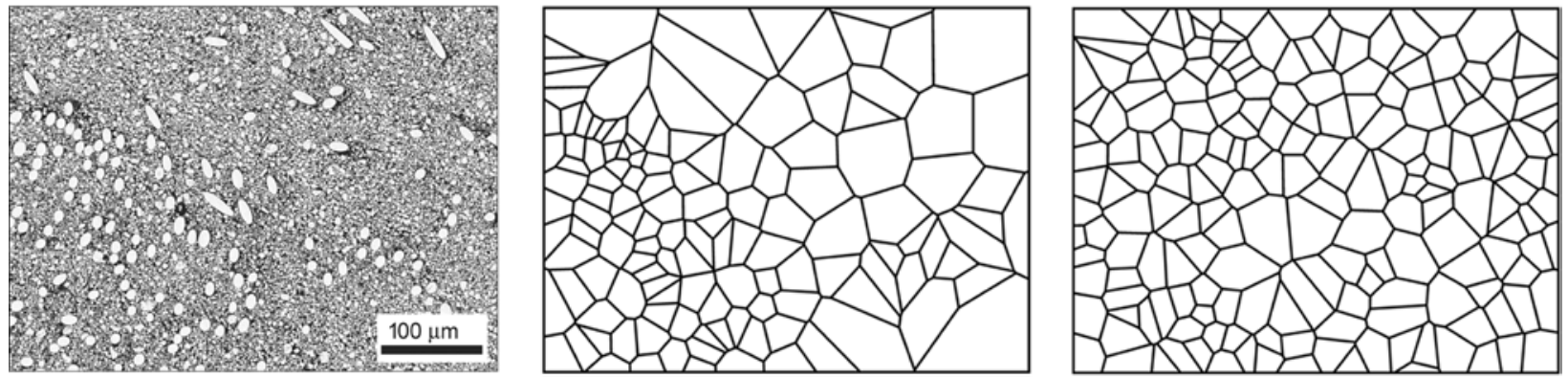

b)
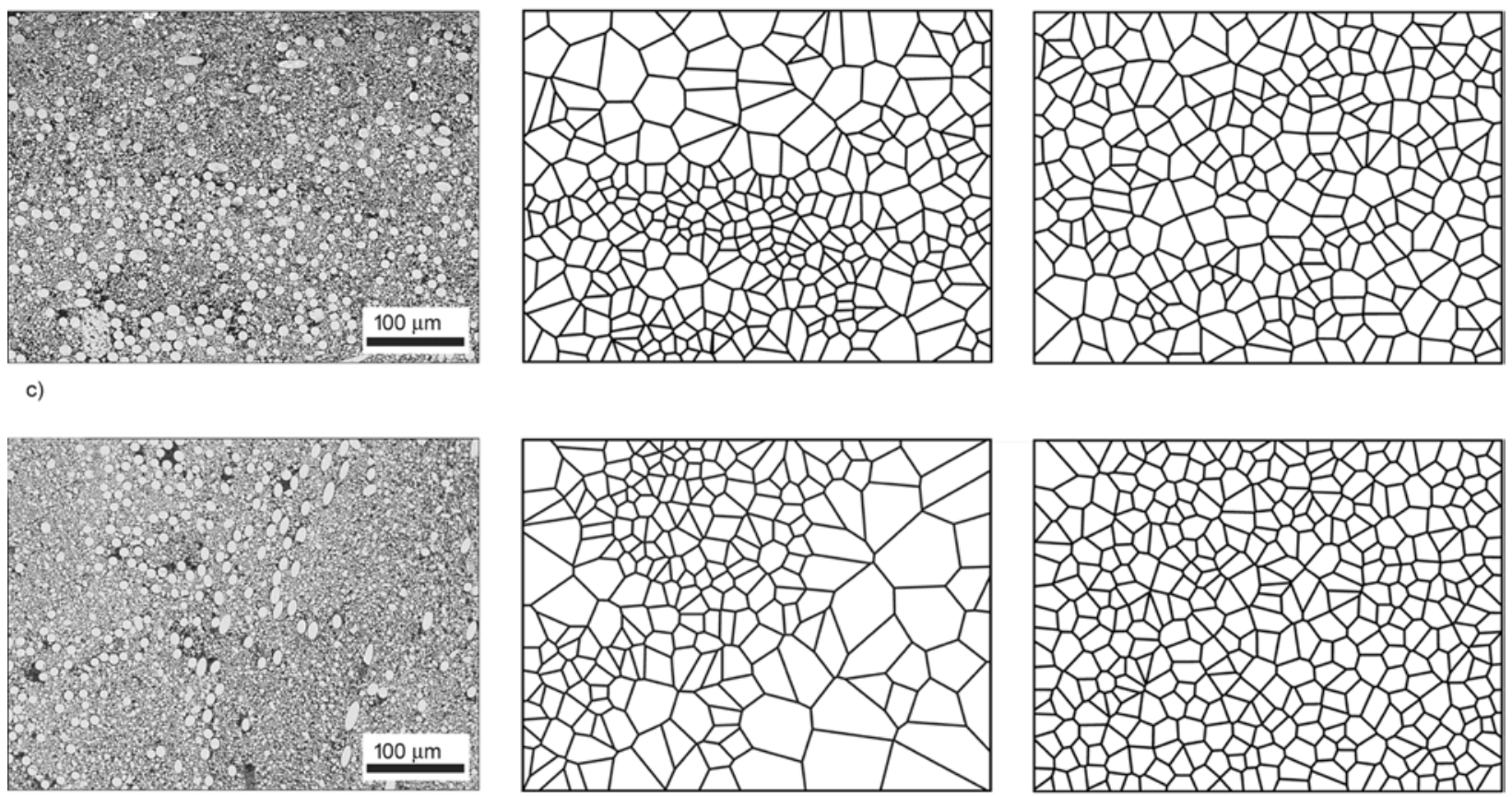

d)

Figure 3. SEM micrographs of polished cross-sections of the BMC composites and the corresponding Voronoi diagrams from the experimental and randomly generated fiber distribution (from area 4): $5 \mathrm{wt} \%$ (a), $10 \mathrm{wt} \%$ (b), $15 \mathrm{wt} \%$ (c), and $20 \mathrm{wt} \%(\mathrm{~d})$ of the EGF

shown in Figure 1, was carried out by RDF analysis. This method was already found to be useful in describing the fiber distribution in fiber-reinforced composites $[17,30]$. In fact, the RDF gives information about the areal density of the fibers at a certain distance and can subsequently indicate the degree of fiber clustering in the microstructure. The results are shown in Figure 4 and include all 9 analyzed segments for all composites. The general shape of the experimental and modeled RDFs is similar. At distances below the fiber diameter the RDFs are equal to 0 , since the fibers cannot interpenetrate each other. After the distance of closest approach of about $10 \mu \mathrm{m}$, the $g(r)$ increases rapidly above 1 and later 

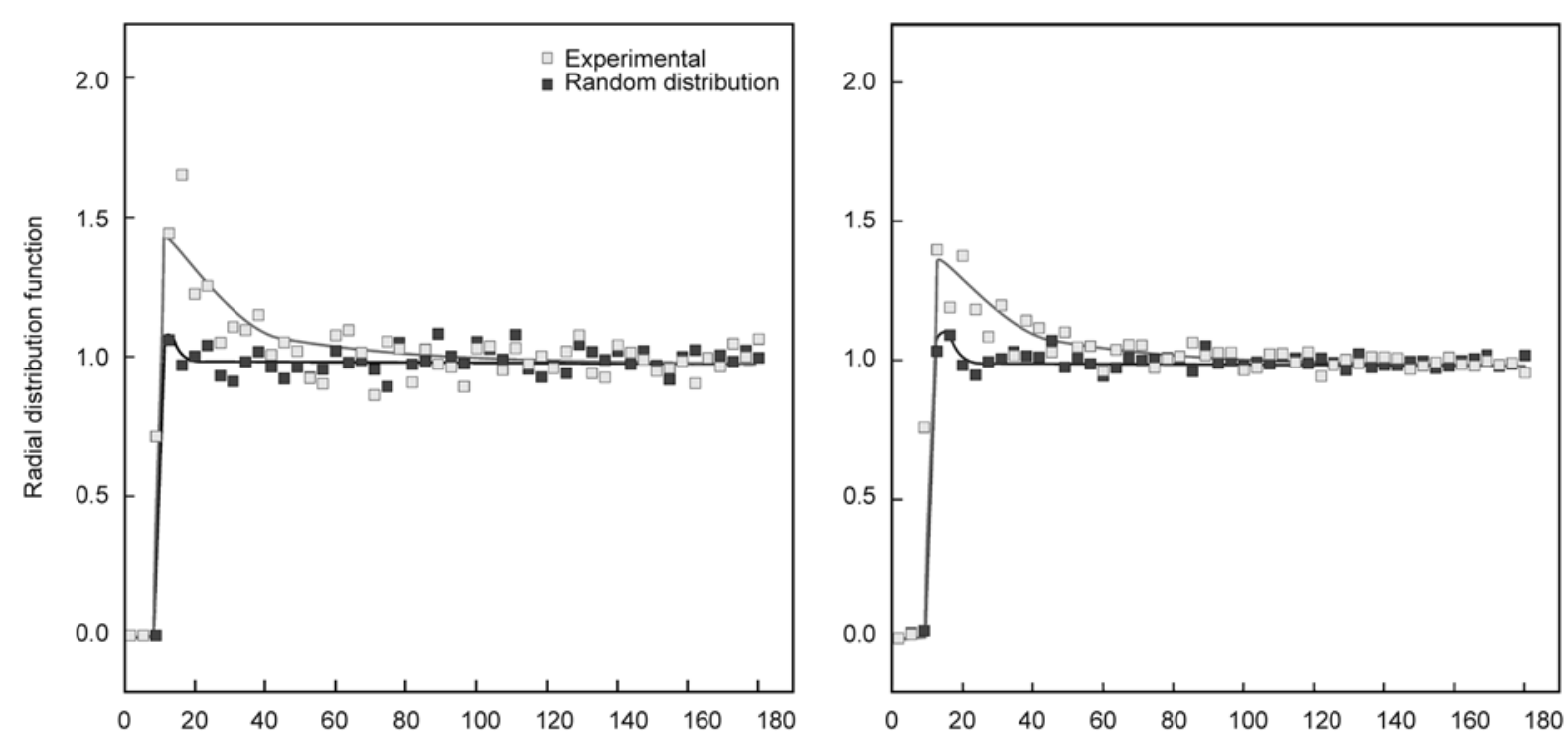

a)
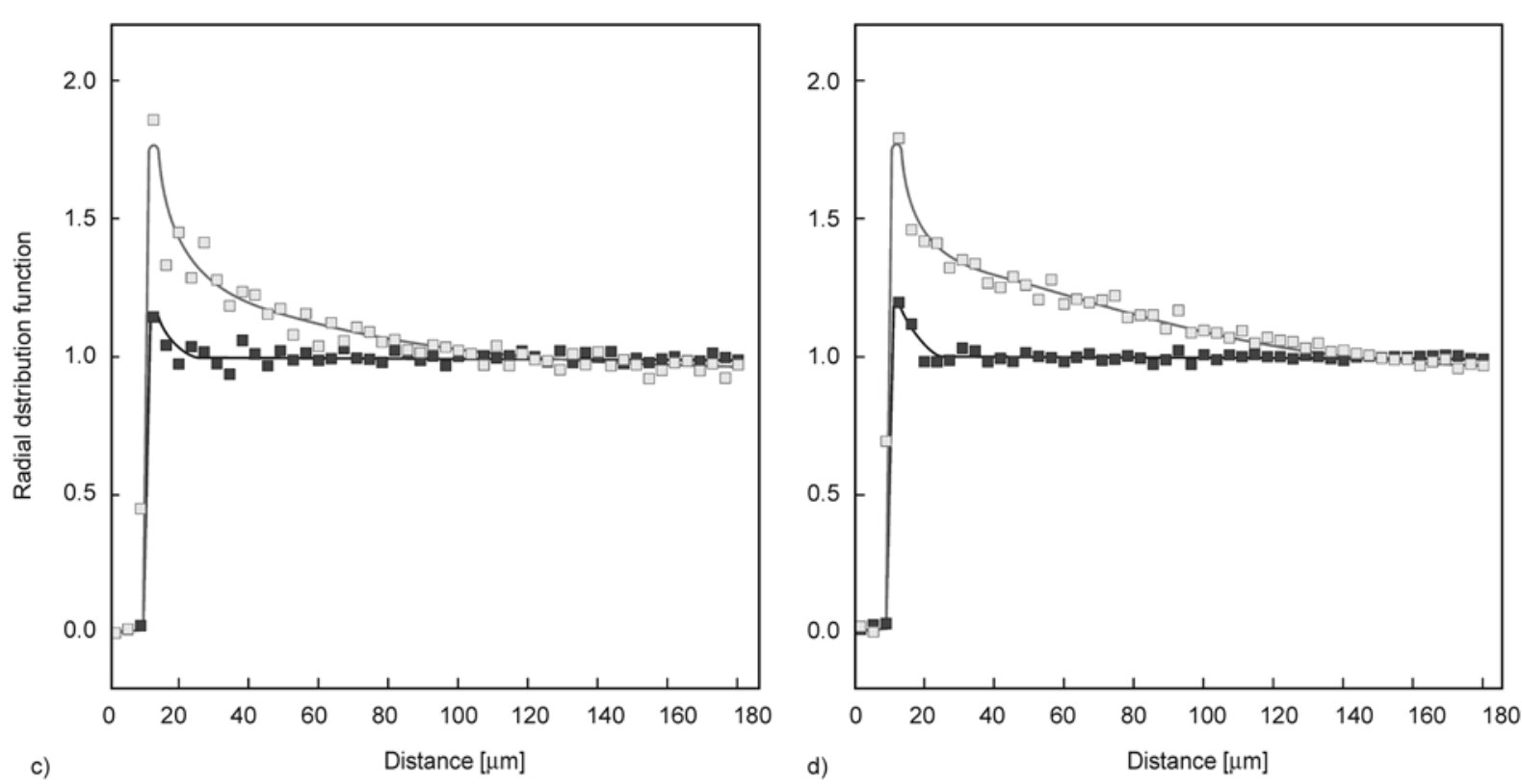

Figure 4. The RDFs of the experimental and randomly generated fiber patterns for all the BMC composites: a) $5 \mathrm{wt} \%$, b) $10 \mathrm{wt} \%$, c) $15 \mathrm{wt} \%$ and d) $20 \mathrm{wt} \%$ of EGF

decays to a value of 1 at large distances. The peak at the distance of closest approach shows that the density of the fibers at this distance is higher than the average density and can be connected to the clustering of the fibers.

Obviously, the experimental RDF values are higher than in the modeled RDFs in all the composites, especially at distances below $40 \mu \mathrm{m}$. The difference is even more noticeable at higher weight fractions of the EGF (15 and $20 \mathrm{wt} \%$ of EGF), indicating additional clustering of the fibers, which can be assigned to their increased number. As an example of such fiber-clustering, the cross-section microstructure of the segment 5 of the composite with $15 \mathrm{wt} \%$ of EGF is shown in Figure 5.

Hence, the values of the experimental fiber distribution are approaching the values of the ideal distribution with increasing distances, and they are reached sooner at a lower EGF weight content, i.e., at 5 and $10 \mathrm{wt} \%$ of EGF. Based on these results, it can be concluded that the distribution of fibers in the composites with 5 and $10 \mathrm{wt} \%$ of EGF most closely approximates to the values extracted from the random distributions.

The relative frequencies of the Voronoi-polygon areas from the experimental and simulated microstruc- 


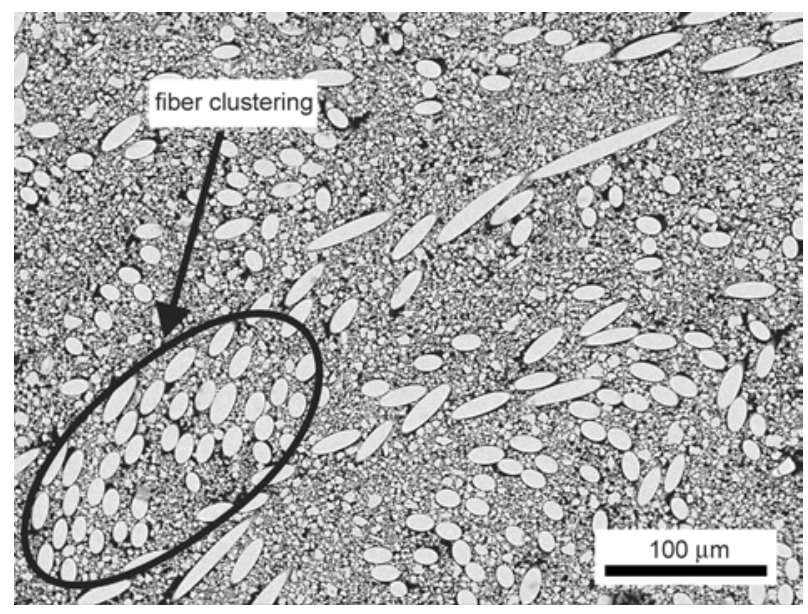

Figure 5. Cross section microstructure of the segment 5 of the composite with $15 \mathrm{wt} \%$ of EGF where fiber clustering is observed

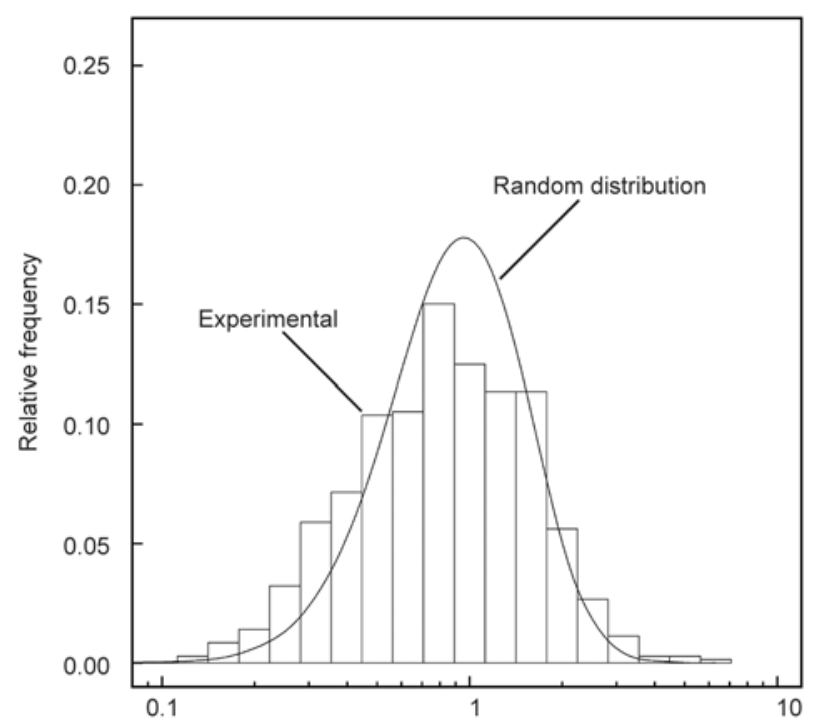

a)

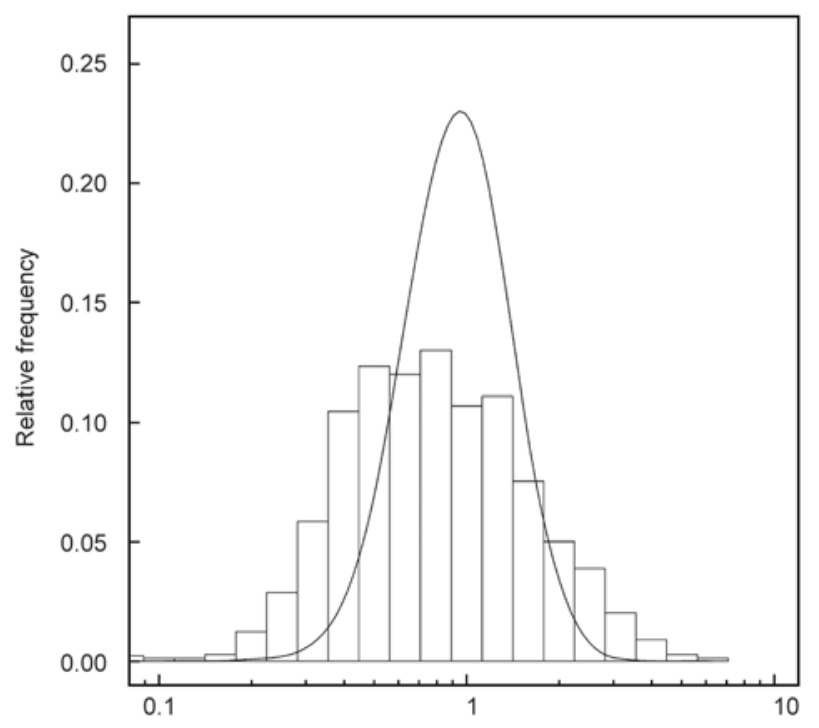

c)

Relative polygon area tures are shown in Figure 6. In the case of the composite with $5 \mathrm{wt} \%$ of EGF the distribution is quite broad due to the larger number of smaller and larger polygons. This is a consequence of local areas with only resin and mineral filler that occur in the composite microstructure at lower weight fractions of EGF. Therefore, the spatial distances between the fibers are quite large in some areas, resulting in larger areas for the Voronoi polygons. Here, the experimental and modeled distributions of EGF are very close. However, the coincidence of the experimental and random distribution plots does not imply that such a composition is the best from the viewpoint of mechanical performance, since the mechanical properties depend on both the content

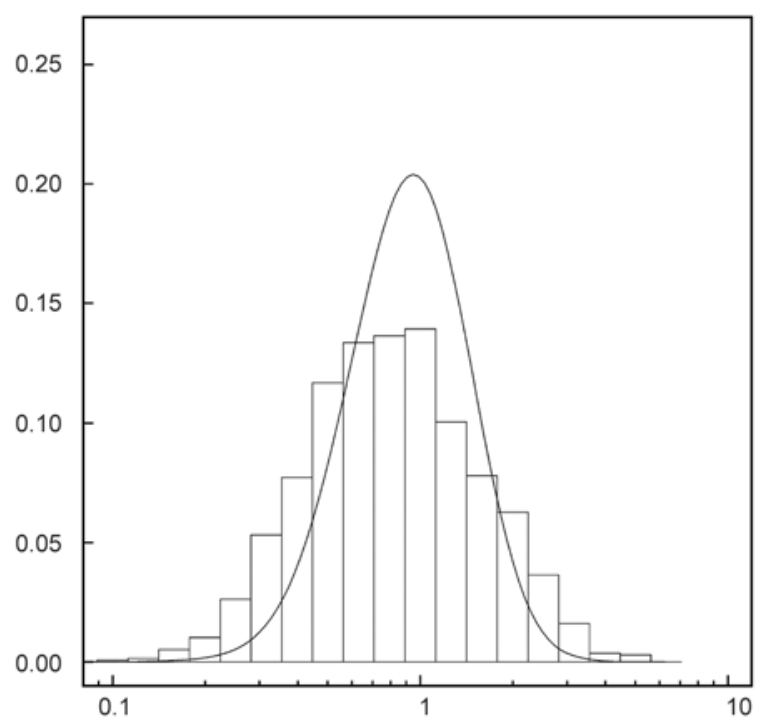

b)

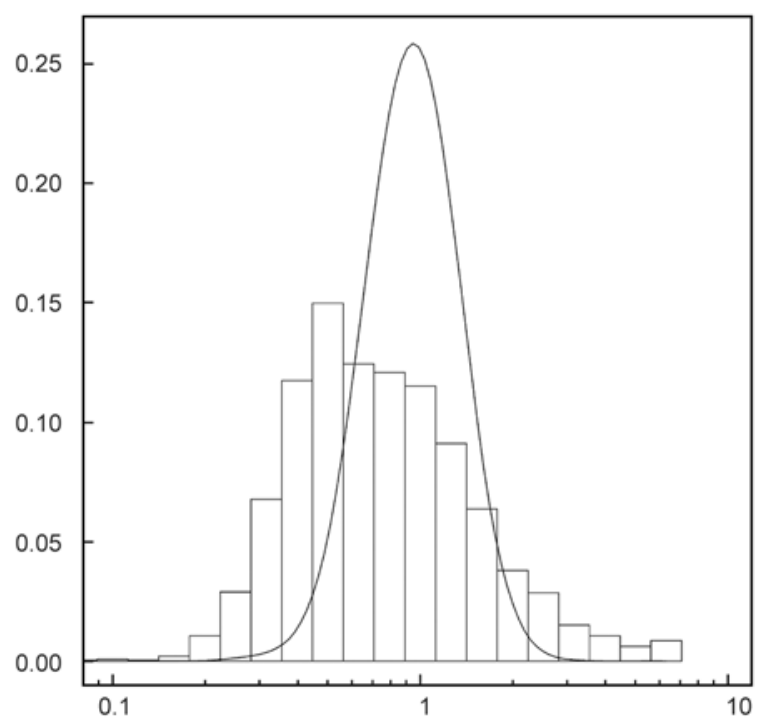

d)

Relative polygon area

Figure 6. Histograms of the relative polygon areas of all the BMC composites extracted from the Voronoi diagrams of the experimental and randomly generated fiber-distribution pattern: a) $5 \mathrm{wt} \%$, b) $10 \mathrm{wt} \%$, c) $15 \mathrm{wt} \%$ and d) $20 \mathrm{wt} \%$ of EGF 
of fibers and their distribution, as discussed below (see section 3.2.).

The histogram of the composite with $10 \mathrm{wt} \%$ of EGF indicates a somewhat narrower distribution of polygons. Such an improved distribution can be mainly related to the increased number of fibers in the PM, i.e., to the decreased number of the local areas filled with resin and mineral filler. Also, the histogram of the ideal random distribution becomes narrower, with the peak shifting to slightly higher values. The divergence of the experimental distribution from the ideal one is even more apparent at higher EGF contents, i.e., at 15 and $20 \mathrm{wt} \%$, which could be primarily the result of clustering in the microstructures, where the distance between the fibers is short and equidistant only locally, rather than across the whole pattern. Due to the closer packing of the fibers at higher weight fractions of the EGF the area sizes of the Voronoi polygons become smaller. This is especially pronounced in the case of the composite with $20 \mathrm{wt} \%$ of EGF, where the peak maximum in the experimental distribution shifts toward a smaller relative polygon area, indicating fiber clustering. In general, one can observe that the histograms of randomly generated distributions are narrower and their peak is higher when the fiber content is increased. This observation suggests more even distributions at higher fractions of fibers, which is the consequence of a smaller available space for each fiber. On the other hand, the experimental histograms have a similar width and a shift to smaller polygon areas at higher fiber contents. This behavior shows that in the experimental samples, the clustering, which counteracts the evenness, is increasingly pronounced with increasing fiber content. This observation is very much in line with the results from the RDF analysis. This was also confirmed with a stereological analysis. The elongation parameter, expressed as the ratio of the longest and shortest axis of the fiber crosssection, and is equal to 1 for a perfect circle, is shown in Figure 7. The results are presented for all 9 segments of an individual composite. The higher the weight percent of EGF, the more spread out the data points are over a wider range of values, meaning that almost round, to highly elongated, intersections of fibers are found in the cross-section of the microstructure of the sample with $20 \mathrm{wt} \%$ of EGF, with the elongation parameter values in some fibers even exceeding 25. By increasing the glass-fiber content, the mean elongation parameter of the EGF increases

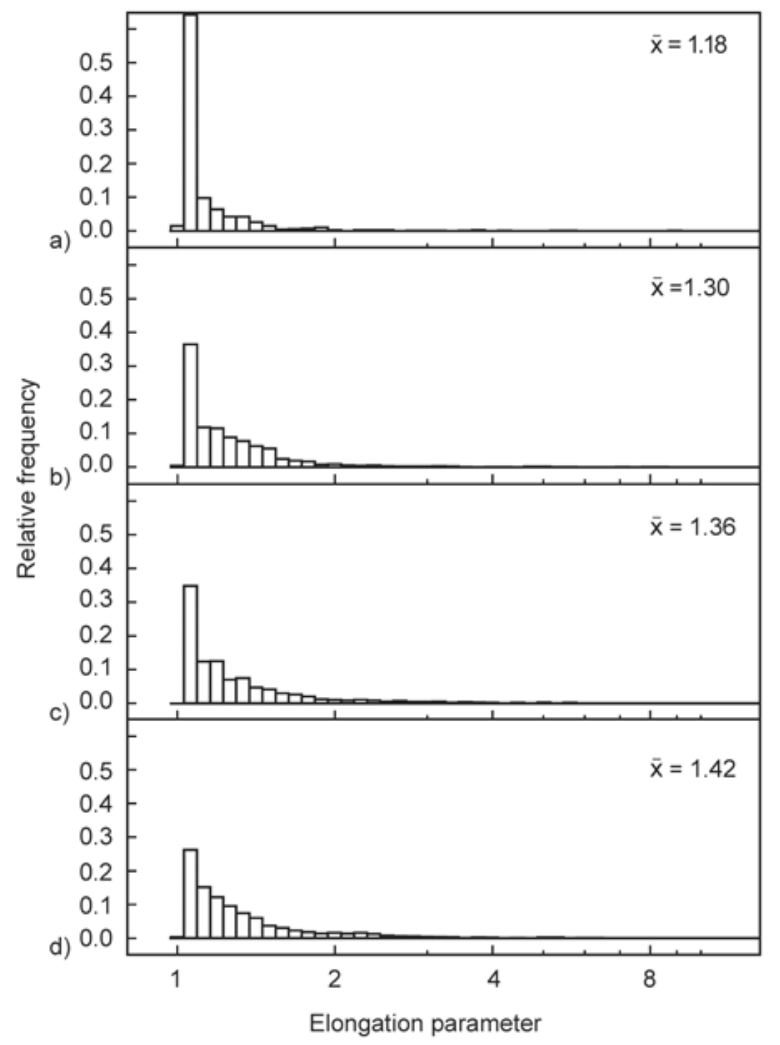

Figure 7. Distribution and mean values of the elongation parameter in the cross-section microstructures of the composites: a) $5 \mathrm{wt} \%$, b) $10 \mathrm{wt} \%$, c) $15 \mathrm{wt} \%$ and d) $20 \mathrm{wt} \%$ of EGF

from 1.18 to 1.42 for 5 and $20 \mathrm{wt} \%$ of EGF, respectively.

\subsection{Mechanical properties}

\subsubsection{Flexural strength}

The flexural strength, the standard deviation, the relative standard deviation and the flexural modulus for the BMC composites with different EGF contents are presented in Table 2. It is evident that with increasing glass-fiber content the flexural strength increases from 73.4 to $108.8 \mathrm{MPa}$ for the samples with $5 \mathrm{wt} \%$ of EGF and $10 \mathrm{wt} \%$ of EGF, respectively, reaching a maximum value of $121.0 \mathrm{MPa}$ for the composite with $15 \mathrm{wt} \%$ of EGF, thus showing the effectiveness of the reinforcement given by the EGF. With a further increase of the EGF content the flexural strength decreases to $95.8 \mathrm{MPa}$, i.e., for the sample with $20 \mathrm{wt} \%$ of EGF. However, an increase in the flexural strength, to some degree, of the composites was expected. It seems that at higher weight fractions, the number of contacts between the fibers increases, thus it may affect the effective stress transfer in the material and, consequently cause a flexural strength reduction that we observed in our samples. 
The clustering of fibers and the increased fiberfiber interactions at higher fiber contents were also observed in the SEM micrographs (see Figure 5). This is especially apparent in the composite with $20 \mathrm{wt} \%$ of EGF, where the flexural strength was reduced. The results of the flexural strength measurements can be related to the degree of fiber-distribution homogeneity in the individual composites, as previously indicated in Figure 5 by the RDF. The values of the relative standard deviation for the composites with 15 and $20 \mathrm{wt} \%$ of EGF indicate an uneven load capacity, since they are higher (RSD > $10 \%$ ) than for the composites with a lower EGF fraction. The composite with $10 \mathrm{wt} \%$ of EGF has the smallest value of the relative standard deviation, i.e., $3.3 \%$, (see Table 2) suggesting the most uniform stress transfer between the fibers. The presence of differently oriented fibers in the microstructure, as evidenced by the increased elongation factor (see Figure 7), could locally increase the stress, which could affect the interfacial de-bonding and brittle-matrix cracking and could be responsible for the less effective mechanical performance of the composite material. Furthermore, it is important to note that at lower weight fractions of the EGF, below $10 \mathrm{wt} \%$, there are not enough fibers to reinforce the $\mathrm{PM}$ and to achieve sufficient mechanical strength. Flexural modulus of all BMC composites is increasing with fiber content from 15.2 to $16.9 \mathrm{GPa}$ for 5 and $20 \mathrm{wt} \%$ of EGF, respectively.

\subsubsection{Dynamic mechanical analysis}

In order to study the mechanical response of the composites with different weight portions of EGF, a

Table 2. Flexural strength, $\left(\sigma_{\mathrm{f}}\right)$, standard deviation, $(S D)$, relative standard deviation, $(R S D)$ and flexural modulus, $\left(E_{\mathrm{f}}\right)$, of the BMC composites. In the bottom part of the table is listed the coefficient of reinforcement $(C)$ obtained from the storage modulus (DMA analysis) and $T_{\mathrm{g}}$ values of all BMC composites determined from the $\tan \sigma$ curves

\begin{tabular}{|l|c|c|c|c|c|}
\hline \multirow{2}{*}{} & \multicolumn{5}{|c|}{ Weight content of EGF } \\
[wt\%] \\
\cline { 2 - 6 } & $\mathbf{0}$ & $\mathbf{5}$ & $\mathbf{1 0}$ & $\mathbf{1 5}$ & $\mathbf{2 0}$ \\
\hline$\sigma_{\mathrm{f}}[\mathrm{MPa}]$ & & 73 & 109 & 121 & 96 \\
\hline$S D$ & & 5.4 & 3.6 & 15.3 & 9.8 \\
\hline$R S D[\%]$ & \multicolumn{5}{|c|}{ DMA } \\
\hline$E_{\mathrm{f}}[\mathrm{GPa}]$ & 7.4 & 3.3 & 12.6 & 10.2 \\
\hline & 15.2 & 15.4 & 16.7 & 16.9 \\
\hline$C$ & \multicolumn{7}{|c|}{0.85} & 0.79 & 0.87 \\
\hline$T_{\mathrm{g}}\left[{ }^{\circ} \mathrm{C}\right]$ & 99 & 101 & 94 & 91 & 113 \\
\hline
\end{tabular}

DMA was performed with the aim being to correlate the obtained results with the microstructural analyses. The composite without EGF ( $0 \mathrm{wt} \% \mathrm{EGF}$, $79 \mathrm{wt} \% \mathrm{CaCO}_{3}$ ) was additionally analyzed to obtain a better insight into how the viscoelastic properties change with the fiber loading. The temperature dependence of the storage modulus $\left(E^{\prime}\right)$, the loss modulus $\left(E^{\prime \prime}\right)$ and the damping behavior $(\tan \delta)$ for all the BMC composites are shown in Figure 8. Evidently, the composites' storage moduli increased with the addition of the EGF to the host PM across the entire temperature range due to the increase in the material stiffness. The coefficient of reinforcement, calculated using Equation (6):

$$
C=\frac{\left(\frac{E_{\mathrm{G}}^{\prime}}{E_{\mathrm{R}}^{\prime}}\right)_{\text {composite }}}{\left(\frac{E_{\mathrm{G}}^{\prime \prime}}{E_{\mathrm{R}}^{\prime}}\right)_{\mathrm{PM}+\text { minereal filter }}}
$$

where $E_{\mathrm{G}}^{\prime}$ and $E_{\mathrm{R}}^{\prime}$ are the storage-modulus values in the glassy and rubbery regions, respectively, was used to evaluate the extent of the reinforcing action. According to the theory, the lower the value of $C$ is, the greater is the reinforcement. The calculations (see Table 2) revealed that the sample with $15 \mathrm{wt} \%$ of EGF has the lowest $C$ coefficient and therefore the highest reinforcement.

The temperature dependence of the loss modulus is shown in Figure 8b). Below the glass-transition temperature $\left(T_{\mathrm{g}}\right)$ the loss modulus increases with increasing EGF content up to $15 \mathrm{wt} \%$, which is indicative of better packing of the fibers in the PM and their effective coupling. The $T_{\mathrm{g}}$, when determined as the peak value of the loss modulus curves, is about 85 $96^{\circ} \mathrm{C}$ for all the composites. Above the $T_{\mathrm{g}}$, a sharp decrease is observed, which is assigned to the enhanced movement of the polymer chains. In the case of the composite with $20 \mathrm{wt} \%$ of EGF the deviation from the trend is attributed to the increased fiber-fiber interactions that can lead to faults in the material and deterioration of the mechanical properties.

As shown in Figure 8c), the damping factor for all the composites $(\tan \delta)$ increases with the increasing temperature. All the curves reach a peak in the temperature range from 91 to $113^{\circ} \mathrm{C}\left(T_{\mathrm{g}}\right)$, see Table 2 According to [23], the incorporation of fibers in the PM should lower the $\tan \delta$ peak due to the greater restriction of the polymer chains caused by the presence of fibers. In contrast to the literature, here the 


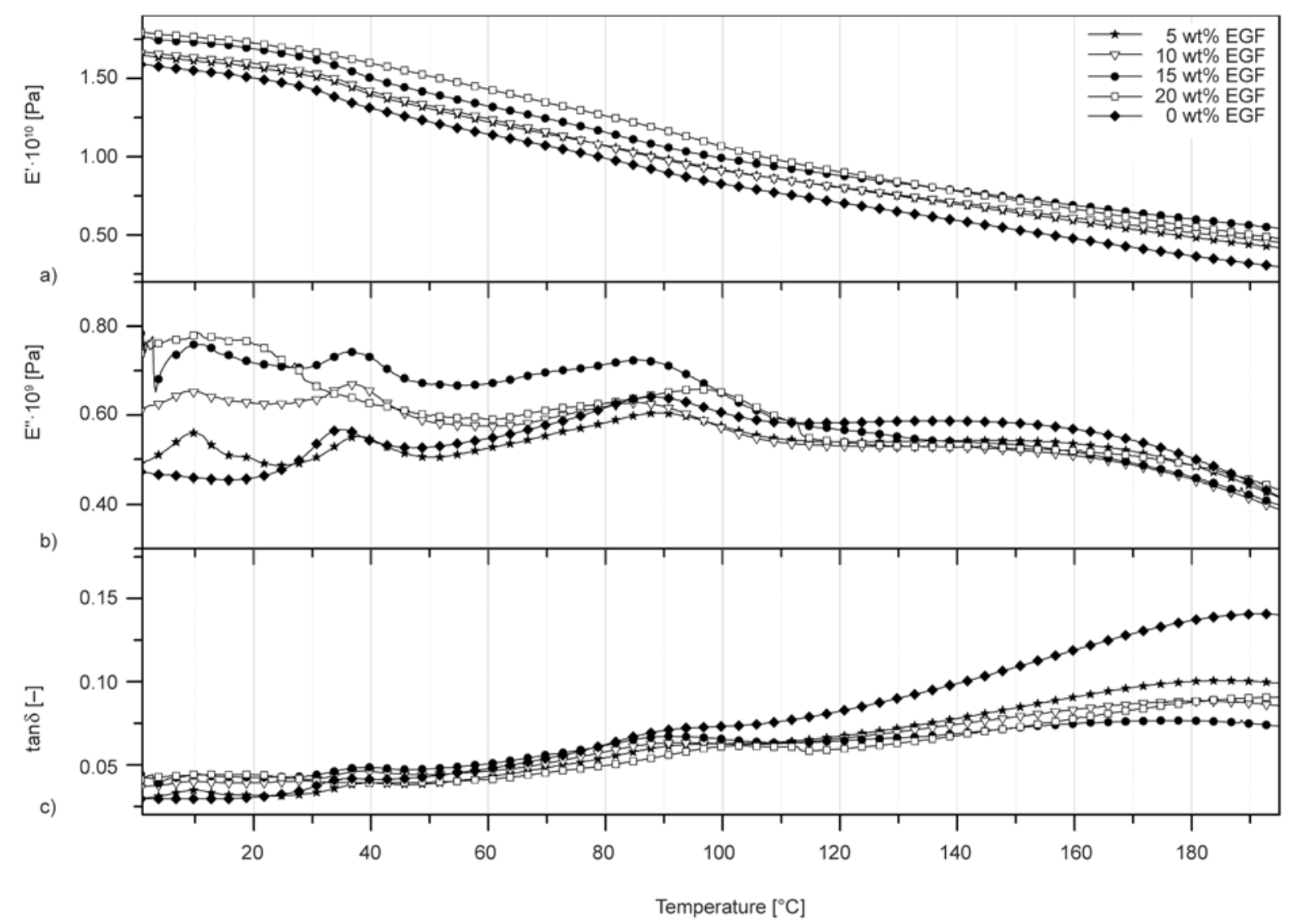

Figure 8. a) storage modulus $\left(E^{\prime}\right)$, b) loss modulus $\left(E^{\prime \prime}\right)$ and c) damping behavior $(\tan \delta)$ for all the BMC composites

opposite was observed for the composites with 15 , 10 and $5 \mathrm{wt} \%$ of EGF, which is probably influenced by the presence of $\mathrm{CaCO}_{3}$ mineral filler.

\section{Conclusions}

Different characterization techniques were used to analyze the EGF distribution in the polymer matrix in order to obtain a better insight into how the degree of the fibers' order/disorder and their orientation influence the mechanical properties of fiberreinforced composites. Methodologies and computational techniques such as the Dirichlet tessellation and the RDF were considered during a quantitative characterization of the composites, where a set of criteria was used to determine whether the simulated microstructures are, to some degree, equivalent to the experimental observations and to link the microstructural changes with the composite properties, i.e., the flexural strength, storage modulus, loss modulus and damping behavior.

According to the results of the RDF analyses the experimental fiber distribution approaches the ideal fiber distribution with increasing distances and is reached more quickly at low weight fractions of the
EGF. It was shown that the fiber distributions in the composites with 5 and $10 \mathrm{wt} \%$ EGF most closely approximate the values of ideal random fiber distributions. Consistent results were obtained from histograms of the relative frequency of the Voronoi diagrams, where the divergence of the experimental fiber distribution from the ideal one is more pronounced at higher fiber contents, i.e., above $15 \mathrm{wt} \%$. Such results indicate that for higher fiber contents the fibers are clustered together, resulting in areas where the distances between the fibers are only locally equidistant. Such a microstructural inhomogeneity then affects and deteriorates the mechanical properties: increased fiber-fiber interactions lead to a less effective stress transfer between the fibers. The flexural strength of the composites increased with increasing EGF content, but only up to a certain point. The highest value was measured for the composite with $15 \mathrm{wt} \%$ of EGF. The coefficient of reinforcement showed an optimum value for the composite with $15 \mathrm{wt} \%$ of EGF. We can conclude that the right balance of properties, i.e., a high degree of fiber distribution homogeneity and a suitable mechanical performance can be achieved by tailoring the EGF con- 
tent for a selected composite formulation. Furthermore, such quantitative analysis of the microstructure of BMC composites could be applied for obtaining information about the mechanical response of complex parts of BMC products.

\section{Acknowledgements}

BB gratefully acknowledges the financial support of the Ministry for Education, Science and Sport of the Republic of Slovenia and the European Commission through the European Social Fund. BM and KV acknowledge the financial support of the Slovenian Research Agency (research program P2-0105), and EŽ and MH (program P2-0145).

\section{References}

[1] Burns R.: Polyester molding compounds. Marcel Dekker, New York (1982).

[2] Monk J. F.: Thermosetting plastics: Moulding materials and processes. Longman, Harlow (1997).

[3] Harper L. T., Turner T. A., Warrior N. A., Dahl J. S., Rudd C. D.: Characterisation of random carbon fibre composites from a directed fibre preforming process: Analysis of microstructural parameters. Composites Part A: Applied Science and Manufacturing, 37, 21362147 (2006). DOI: 10.1016/j.compositesa.2005.11.014

[4] Sreenivasan V. S., Ravindran D., Manikandan V., Narayanasamy R.: Mechanical properties of randomly oriented short Sansevieria cylindrica fibre/polyester composites. Materials and Design, 32, 2444-2455 (2011).

DOI: $10.1016 /$ j.matdes.2010.11.042

[5] Guild F. J., Summerscales J.: Microstructural image analysis applied to fibre composite materials: A review. Composites, 24, 383-393 (1993).

DOI: 10.1016/0010-4361(93)90246-5

[6] Avérous L., Quantin J-C., Lafon D., Crespy A.: Morphological determinations of fiber composites. Microscopy Microanalysis Microstructures, 7, 433-439 (1996).

DOI: $10.1051 / \mathrm{mmm}: 1996142$

[7] Zangenberg J., Larsen J. B., Østergaard R. C., Brøndsted P.: Methodology for characterisation of glass fibre composite architecture. Plastics, Rubber and Composites, 41, 187-193 (2012).

DOI: $10.1179 / 1743289811$ Y.0000000067

[8] Voronoi G.: Nouvelles applications des paramètres continus à la théorie des formes quadratiques. Deuxième mémoire. Recherches sur les parallélloèdres primitifs. (in French). Journal für die reine und angewandte Mathematik, 134, 198-287 (1908).
[9] Voronoi G.: Nouvelles applications des paramètres continus à théorie des formes quadratiques. Deuxième mémoire. Recherches sur les paralléloèdres primitifs. (in French). Journal für die reine und angewandte Mathematik, 136, 67-182 (1909).

[10] Brostow W., Castaño V. M.: Voronoi polyhedra as a tool for dealing with spatial structures of amorphous solids, liquids and gases. Journal of Materials Education, 21, 297-304 (1999).

[11] Medvedev N. N., Geiger A., Brostow W.: Distinguishing liquids from amorphous solids: Percolation analysis on the Voronoi network. Journal of Chemical Physics, 93, 8337-8342 (1990).

DOI: $10.1063 / 1.459711$

[12] Kalogeras I. M., Hagg Lobland H. E.: The nature of the glassy state: Structure and transitions. Journal of Materials Education, 34, 69-94 (2012).

[13] Heijman M. J. G. W., Benes N. E., ten Elshof J. E., Verweij H.: Quantitative analysis of the microstructural homogeneity of zirconia-toughened alumina composites. Materials Research Bulletin, 37, 141-149 (2002). DOI: 10.1016/S0025-5408(01)00806-6

[14] Trefalt G., Benčan A., Kamplet M., Malič B., Seo Y., Webber K. G.: Evaluation of the homogeneity in $\mathrm{Pb}(\mathrm{Zr}, \mathrm{Ti}) \mathrm{O}_{3}$-zirconia composites prepared by the hetero-agglomeration of precursors using the Voronoidiagram approach. Journal of the European Ceramic Society, 34, 669-675 (2014).

DOI: 10.1016/j.jeurceramsoc.2013.09.014

[15] Summerscales J., Guild F. J., Pearce N. R. L., Russell P. M.: Voronoi cells, fractal dimensions and fibre composites. Journal of Microscopy, 201, 153-162 (2001). DOI: $10.1046 / \mathrm{j} .1365-2818.2001 .00841 . \mathrm{x}$

[16] Sun Z., Zhao X., Ma J.: Characterization of microstructures in sisal fiber composites by Voronoi diagram. Journal of Reinforced Plastics and Composites, 32, 16-22 (2012). DOI: $10.1177 / 0731684412461191$

[17] Ghosh S., Nowak Z., Lee K.: Quantitative characterization and modeling of composite microstructures by Voronoi cells. Acta Materialia, 45, 2215-2234 (1997). DOI: $10.1016 / \mathrm{S} 1359-6454(96) 00365-5$

[18] Pyrz R., Bochenek B.: Topological disorder of microstructure and its relation to the stress field. International Journal of Solids and Structures, 35, 2413-2427 (1998). DOI: 10.1016/S0020-7683(97)00140-6

[19] Pinheiro A., Mano J. F.: Study of the glass transition on viscous-forming and powder materials using dynamic mechanical analysis. Polymer Testing, 28, 89-95 (2009). DOI: $10.1016 /$ j.polymertesting.2008.11.008

[20] Budai Z., Sulyok Z., Vargha V.: Glass-fibre reinforced composite materials based on unsaturated polyester resins. Journal of Thermal Analysis and Calorimetry, 109, 1533-1544 (2012). DOI: $10.1007 / \mathrm{s} 10973-011-2069-5$ 
[21] Devi L. U., Bhagawan S. S., Thomas S.: Dynamic mechanical analysis of pineapple leaf/glass hybrid fiber reinforced polyester composites. Polymer Composites, 31, 956-965 (2010).

DOI: $10.1002 /$ pc. 20880

[22] Idicula M., Malhotra S. K., Joseph K., Thomas S.: Dynamic mechanical analysis of randomly oriented intimately mixed short banana/sisal hybrid fibre reinforced polyester composites. Composites Science and Technology, 65, 1077-1087 (2005).

DOI: $10.1016 /$ j.compscitech.2004.10.023

[23] Romanzini D., Lavoratti A., Ornaghi Jr H. L., Amico S. C., Zattera A. J.: Influence of fiber content on the mechanical and dynamic mechanical properties of glass/ ramie polymer composites. Materials and Design, 47, 9-15 (2013).

DOI: $10.1016 /$ j.matdes.2012.12.029

[24] Almeida Júnior J. H. S., Ornaghi Júnior H. L., Amico S. C., Amado F. D. R.: Study of hybrid intralaminate curaua/glass composites. Materials and Design, 42, 111-117 (2012).

DOI: $10.1016 /$ j.matdes.2012.05.044
[25] ISO 3167: Plastics. Multipurpose test specimens (2002).

[26] Járai-Szabó F., Néda Z.: On the size distribution of Poisson Voronoi cells. Physica A: Statistical Mechanics and its Applications, 385, 518-526 (2007). DOI: $10.1016 /$ j.physa.2007.07.063

[27] Allen M. P., Tildesley D. J.: Computer simulation of liquids. Clarendon Press, Oxford (1989).

[28] Frenkel D., Smit B.: Understanding molecular simulation: From algorithms to applications. Academic Press, San Diego (2001).

[29] ISO 178: Plastics. Determination of flexural properties (2003).

[30] Everett R. K.: Quantification of random fiber arrangements using a radial distribution function approach. Journal of Composite Materials, 30, 748-758 (1996). DOI: $10.1177 / 002199839603000606$ 